\title{
Magnetism of a tetrahedral cluster spin-chain
}

\author{
Wolfram Brenig \\ Institut für Theoretische Physik, Technische Universität Braunschweig, 38106 Braunschweig, Germany \\ Klaus W. Becker \\ Institut für Theoretische Physik, Technische Universität Dresden, 01062 Dresden, Germany
}

Dedicated to Professor Erwin Müller-Hartmann on the occasion of his $60^{\text {th }}$ birthday.

(October 31, 2018)

We discuss the magnetic properties of a dimerized and completely frustrated tetrahedral spin-1/2 chain. Using a combination of exact diagonalization and bond-operator theory the quantum phase diagram is shown to incorporate a singlet-product, a dimer, and a Haldane phase. In addition we consider one-, and two-triplet excitations in the dimer phase and evaluate the magnetic Raman cross section which is found to be strongly renormalized by the presence of a two-triplet bound state. The link to a novel tellurate materials is clarified.

PACS numbers: 75.10.Jm, 75.50.Ee, 75.40.-s, 78.30.-j

\section{INTRODUCTION}

Low-dimensional quantum-magnetism has received considerable interest recently due to the discovery of numerous novel materials with spin- $\frac{1}{2}$ moments arranged in chain, ladder, and depleted planar structures. Many of these materials exhibit unconventional magnetic phases due to dimerization and frustration of antiferromagnetic exchange. Particular effort 1 has been devoted to systems like $\mathrm{SrCu}_{2}\left(\mathrm{BO}_{3}\right)_{2}$, 3 , which display a complete frustration of the magnetic exchange as in the two-dimensional Shastry-Sutherland modelt. In one dimension complete frustration can occur in two-leg spin-ladders if an additional cross-wise exchange is included as depicted in fig. 11 which resembles a chain of edge sharing tetrahedra. For $J_{1}=J_{3}$ such tetrahedral ladders have been investigated in the past 5 . Very recently, tellurates of type $\mathrm{Cu}_{2} \mathrm{Te}_{2} \mathrm{O}_{5} \mathrm{X}_{2}$ with $\mathrm{X}=\mathrm{Cl}$, Br have been identified as a new class of spin-1/2 tetrahedral-cluster compounds 8 . Bulk thermodynamic data have been analyzed in the limit of isolated tetrahedra 6 . Raman spectroscopy, however indicates a substantial inter-tetrahedral coupling along the c-axis direction of $\mathrm{Cu}_{2} \mathrm{Te}_{2} \mathrm{O}_{5} \mathrm{X}_{2} \mathrm{9}$. In this direction the exchange topology is likely to be analogous to that of fig. 1 with $J_{1} \neq J_{3}$. From a materials perspective it is an open question if the magnetism of the tellurates can be understood in terms of a dimerized tetrahedral spin-ladder. From a theoretical point of view, however, the magnetic properties of this model are an interesting issue which forms the motivation for this work.

The paper is organized as follows. In the remainder of this section we discuss the basic properties of the tetrahedral chain hamiltonian. In section two the quantum phase diagram is analyzed. In section three a bondoperator method is applied to the tetrahedral chain and in section four the magnetic Raman cross-section is evaluated.

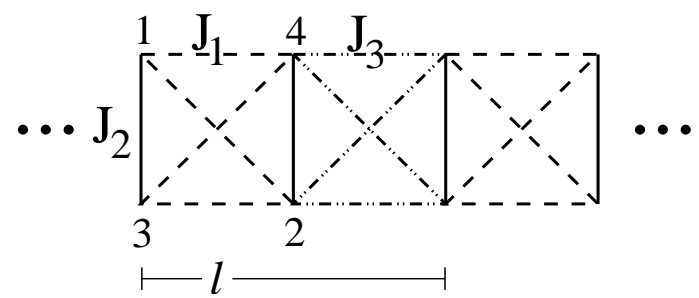

FIG. 1. The tetrahedral cluster-chain. $l$ labels the unit cell containing spin-1/2 moments $\mathbf{s}_{i l}$ at the vertices $i=1, \ldots, 4$.

The hamiltonian of the tetrahedral chain can be written in terms of the total edge-spin operators $\mathbf{T}_{1(2) l}=$ $\mathbf{s}_{1(4) l}+\mathbf{s}_{3(2) l}$ and the dimensionless couplings $b=J_{3} / J_{1}$ and $a=J_{2} / J_{1}$

$$
\frac{H}{J_{1}}=\sum_{l}\left[\mathbf{T}_{1 l} \mathbf{T}_{2 l}+b \mathbf{T}_{2 l} \mathbf{T}_{1 l+1}+\frac{a}{2}\left(\mathbf{T}_{1 l}^{2}+\mathbf{T}_{2 l}^{2}\right)-\frac{3 a}{2}\right]
$$

This model displays infinitely many local conservation laws: $\left[H, \mathbf{T}_{i(=1,2) l}^{2}\right]=0 ; \forall l, i=1,2$. Therefore, the Hilbert space decomposes into sectors of fixed distributions of edge-spin eigenvalues $T_{i l}=1$ or 0 , each corresponding to a sequences of spin-1 chain-segments intermitted by chain-segments of localized singlets. If $J_{1} \neq J_{3}$ the spin-1 chain-segments are dimerized. In the infinite length, dimerized $S=1$-chain sector, i.e. for $T_{i l}=1 \forall i, l$, the model simplifies to

$$
\frac{H}{J_{1}}=\sum_{l}\left[\mathbf{S}_{1 l} \mathbf{S}_{2 l}+b \mathbf{S}_{2 l} \mathbf{S}_{1 l+1}\right]+\frac{a}{2} D
$$

where $\mathbf{S}_{i l}$ refer to spin-1 operators and $D$ is the number of tetrahedra ('dimers').

The Hilbert space of a single tetrahedron consists of 16 states, i.e., two singlets $\mathcal{S}_{1,2}$, three triplets $\mathcal{T}_{1,2,3}$ and one 


\begin{tabular}{c|c|c|c}
\hline \hline & $T_{1}$ & $T_{2}$ & $E / J_{1}$ \\
\hline $\mathcal{S}_{1}$ & 1 & 1 & $-2+\mathrm{a} / 2$ \\
$\mathcal{S}_{2}$ & 0 & 0 & $-3 \mathrm{a} / 2$ \\
$\mathcal{T}_{1}$ & 1 & 1 & $-1+\mathrm{a} / 2$ \\
$\mathcal{T}_{2,3}$ & 0,1 & 1,0 & $-\mathrm{a} / 2$ \\
$\mathcal{Q}$ & 1 & 1 & $1+\mathrm{a} / 2$ \\
\hline \hline
\end{tabular}

TABLE I. Eigenstates and energies of the tetrahedron. Columns $T_{1}, 2$ refer to corresponding edge-spin quantum number, site index $l$ suppressed.

quintet $\mathcal{Q}$ the energies and $T_{i}$ quantum numbers of which are listed in table I. Johnsson and collaborators have first pointed out that this level scheme implies a singlet to reside within the singlet-triplet gap of the tetrahedron for $1 / 2<a<2$. Moreover the ground state switches from $\mathcal{S}_{1}$ to $\mathcal{S}_{2}$ at $a=1$ suggesting a line of quantum phase transitions in the $(a, b)$-plane for the lattice model.

\section{QUANTUM PHASE DIAGRAM}

In this section we discuss the ground state of the tetrahedral chain. To begin, we note that by a shift of one half of the unit cell, i.e. $\mathbf{T}_{2 l(1 l+1)} \rightarrow \mathbf{T}_{1 l(2 l)}$, model (11) is symmetric under the operation $\left(J_{1}, a, b\right) \rightarrow$ $\left(J_{1} b, a / b, 1 / b\right)$. Therefore, in order to cover the complete parameter space for $a, b>0$ it is sufficient to consider the phase diagram in the range of $a \in[0, \infty]$ and $b \in[0,1]$.

Next we note, that the ground state of (1) will be either in the dimerized $S=1$-chain sector or in a homogeneous product of $\mathcal{S}_{2}$ states only. Inhomogeneous phases consisting of both, $T_{i l}=0$ and $T_{i l}=1$ sites are not allowed for as ground-states. To see this we fix $b$ and assume $a \rightarrow \infty$, in which case the ground state is a pure product of $\mathcal{S}_{2}$-type singlets: $\left|\psi_{0}\right\rangle=\prod_{l}\left|\tilde{s}_{l}\right\rangle$. Next we check for the ground-state energy change $\Delta E(a, b, N)$ upon forming a single connected chain-segment of length $N$ composed out of $T_{i l}=1$-sites within the homogeneous state $\left|\psi_{0}\right\rangle$. To be specific we first assume the chain-segment to consist of $D^{\prime}=N / 2$ tetrahedra in which case

$$
\Delta E\left(a, b, D^{\prime}\right)=D^{\prime}\left[2(a-1)-e\left(b, D^{\prime}\right)\right] .
$$

Here $-e\left(b, D^{\prime}\right)<0$ is the ground-state energy gain per two sites due to the inter-tetrahedral coupling. The main point is that $e\left(b, D^{\prime}\right)$ is a monotonously increasing function 10 of $D^{\prime}$. Therefore the largest critical value $a_{c}=\max \left\{a_{c}\left(D^{\prime}\right)\right\}$ at which the formation of tetrahedra in the $S=1$ sector is favorable, i.e. at which $\Delta E\left(a_{c}\left(D^{\prime}\right), b, D^{\prime}\right)$ turns negative, results for $D^{\prime} \rightarrow \infty$. This implies a single first order quantum phase transition into the infinite-length, dimerized $S=1$-chain sector as a function of decreasing $a$. Similar arguments can be pursued for odd $N$.

In fig. 2 we show the quantum phase diagram. From (3) the first order critical line $a_{c}(b)$ between the infinitelength, dimerized spin-1 chain for $a<a_{c}(b)$ and the

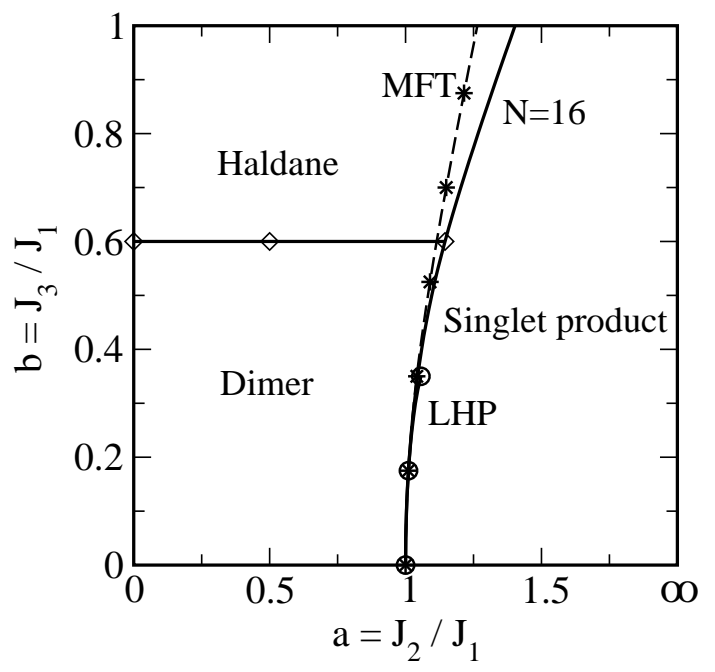

FIG. 2. Quantum phase diagram of the tetrahedral chain. Bare solid line: 1st-oder transition from $\mathrm{ED}$ for $\mathrm{N}=16$ sites and PBC at 41 values of $b \in[0,1]$. The critical value $a_{c}$ at $b=1$ from $\mathrm{ED}$ is $a_{c}^{N=16}(b=1) \simeq 1.40292$. Solid line with diamond markers: 2nd-order Haldane-Dimer transition at $b \simeq 3 / 5$, extrapolated from ED (see fig. 3 and ref. ${ }^{14,15}$ ). Dashes(Solid) line with stared(circled) markers refers to bond-boson mean-field/MFT (Holstein-Primakoff/LHP) approach. LHP terminates at $b=3 / 8$.

$\mathcal{S}_{2}$-type singlet product-state for $a>a_{c}(b)$ is fixed by $a_{c}(b)=1+e(b) / 2$, where $e(b)=\lim _{D^{\prime} \rightarrow \infty} e\left(b, D^{\prime}\right)$. To determine $e(b)$ we have calculated the ground-state energy of dimerized spin-1 chains using exact diagonalization (ED) with periodic boundary conditions (PBC) on up to $N=16$ sites and a bond-boson theory the results of which will be detailed in section III. Regarding the ED the critical value of $a_{c}(b=0)=1$ agrees with ref.6, while $a_{c}^{N=16}(b=1) \simeq 1.403$ agrees with ref.11 and is consistent with an extrapolated value of $a_{c}^{N=\infty}(b=$ $1) \simeq 1.401$ from Density-Matrix-Renormalization-Group (DMRG) calculations121 and ED on 22 sites 13.

Within the dimerized $S=1$-chain sector an additional second-order quantum phase transition exists between the dimer phase for $b<b_{c}$ and the Haldane phase for $b>h_{c}$. This transition has been studied extensively (see eg. 15 and refs, therein), resulting in $b_{c} \simeq 3 / 5$ from DMRG calculation $\$ 14$ and finite-size scaling analysi 15 . However, this transition is not at the focus of our study. In fig. 3 our ED results on the finite-size behavior of the spin gap in the dimerized $S=1$-chain sector are shown as function of $b$, which signals the dimer-Haldane transition and directly reproduces identical data which have been obtained earlier by Kato and Tanaka 14 . Fig. 3 contains additional results for the spin gap from the bond-boson approach which we turn to now. 


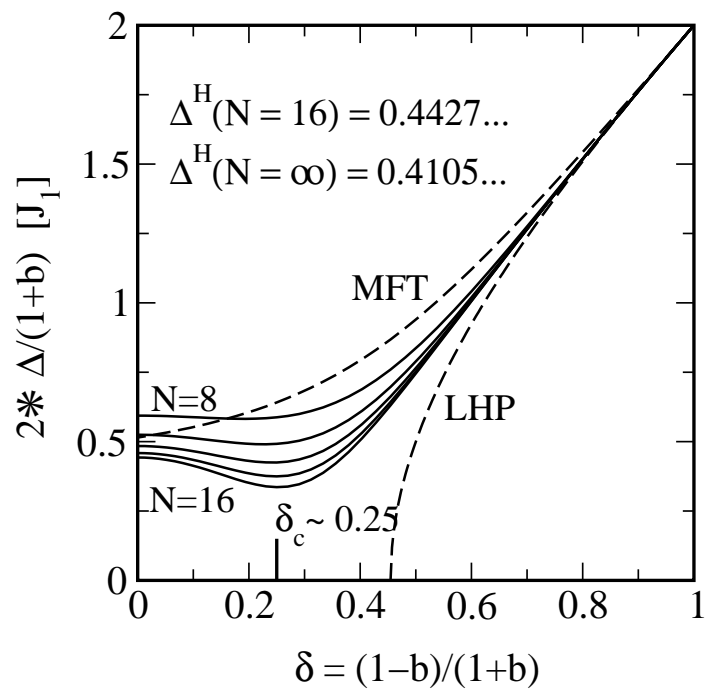

FIG. 3. Solid lines: spin gap $\Delta$ from ED for $N=8,10$, 12,14 , and 16 sites and PBC in the dimerized spin- 1 chain sector at 41 values of the inter-tetrahedral coupling $b \in[0,1]$. Axis have been scaled to allow for a comparison with ref. ${ }^{14}$. $\Delta^{H}$ refers to the spin gap at $b=1$, i.e. the Haldane gap. $\Delta^{H}(N=16)$ as from this work and $\Delta^{H}(N=\infty)$ as from ref. ${ }^{12}$. Upper(lower) dashed line: spin gap from bond-boson mean-field/MFT (Holstein-Primakoff/LHP) approach.

\section{BOND BOSON ANALYSIS}

In this section we detail a mapping of the tetrahedral chain in the dimerized $S=1$-chain sector onto a system of interacting bosons. To this end we adapt the well developed bond-operator method 1621 which has proven to be useful in dimerized spin- $1 / 2$ systems to the present situation. We start by introducing a set of singlet- $\left(s_{l}^{\dagger}\right)$, triplet- $\left(t_{l \alpha}^{\dagger}\right)$, and quintet-bosons $\left(q_{l_{\alpha}}^{\dagger}\right)$ for each tetrahedron at site $l$. These bosons create all states within the multiplets $\mathcal{S}_{1}, \mathcal{T}_{1}$, and $\mathcal{Q}$. The bosons and their corresponding states are listed in table [1. Note, that we have chosen an $x, y, z(z)$ representation for the triplet (quintet) states. Moreover, the site index is not displayed in the table.

To suppress unphysical states the bosons have to fulfill the usual hardcore constraint of no double-occupancy

$$
s_{l}^{\dagger} s_{l}+t_{l \alpha}^{\dagger} t_{l \alpha}+q_{l \alpha}^{\dagger} q_{l \alpha}=1
$$

where doubly appearing Greek indices are to be summed over their respective ranges. After some straightforward algebra we may express the $\alpha=x, y, z$ components of the edge-spins $S_{l 1,2}^{\alpha}$ by

$$
\begin{aligned}
S_{l 1,2}^{\alpha} \hat{=} & \sqrt{\frac{2}{3}}\left( \pm s_{l}^{\dagger} t_{l \alpha} \pm t_{l \alpha}^{\dagger} s_{l}\right)-\frac{i}{2} \varepsilon_{\alpha \beta \gamma} t_{l \beta}^{\dagger} t_{l \gamma} \\
& \pm M_{\alpha \hat{\beta} \hat{\gamma}} t_{l \hat{\beta}}^{\dagger} q_{l \hat{\gamma}} \pm M_{\alpha \hat{\beta} \hat{\gamma}}^{*} q_{l \hat{\gamma}}^{\dagger} t_{l \hat{\beta}}+N_{\alpha \hat{\beta} \hat{\gamma}} q_{l \hat{\beta}}^{\dagger} q_{l \hat{\gamma}} .
\end{aligned}
$$

\begin{tabular}{l|c|r|c}
\hline \hline & BB & $\hat{\alpha}$ & ket \\
\hline $\mathcal{S}_{1}$ & $s^{\dagger}|\rangle$ & 1 & $\frac{1}{\sqrt{3}}(|-+\rangle+|+-\rangle-|00\rangle)$ \\
\hline \multirow{2}{*}{$\mathcal{T}_{1}$} & $t_{x}^{\dagger}|\rangle$ & 2 & $\frac{1}{2}(|0+\rangle-|+0\rangle+|-0\rangle-|0-\rangle)$ \\
& $t_{y}^{\dagger}|\rangle$ & 3 & $\frac{i}{2}(|+0\rangle-|0+\rangle+|-0\rangle-|0-\rangle)$ \\
& $t_{z}^{\dagger}|\rangle$ & 4 & $\frac{1}{\sqrt{2}}(|+-\rangle-|-+\rangle)$ \\
\hline & $q_{2}^{\dagger}|\rangle$ & 5 & $|++\rangle$ \\
& $q_{1}^{\dagger}|\rangle$ & 6 & $\frac{1}{\sqrt{2}}(|+0\rangle+|0+\rangle)$ \\
$\mathcal{Q}$ & $q_{0}^{\dagger}|\rangle$ & 7 & $\frac{1}{\sqrt{6}}(|+-\rangle+2|00\rangle+|-+\rangle)$ \\
& $q_{-1}^{\dagger}|\rangle$ & 8 & $\frac{1}{\sqrt{2}}(|-0\rangle+|0-\rangle)$ \\
& $q_{-2}^{\dagger}|\rangle$ & 9 & $|--\rangle$ \\
\hline \hline
\end{tabular}

TABLE II. Bond-boson (BB) representation of the singlet $\left(\mathcal{S}_{1}\right)$, triplet $\left(\mathcal{T}_{1}\right)$, and quintet $(\mathcal{Q})$ states in the edge-spin $S=1$ sector. |> represents the vacuum. $\hat{\alpha}$ refers to an equivalent running index for each state used to label elements of $M_{\alpha \hat{\beta} \hat{\gamma}}$ and $N_{\alpha \hat{\beta} \hat{\gamma}}$ in (畐). Entries in the ket column refer to $S^{z}$-eigenstates of $S_{1,2}$ of type $\left|S_{1}^{z} S_{2}^{z}\right\rangle$ with $+, 0,-$ denoting $S^{z}=-1,0,+1$.

Since $M_{\alpha \hat{\beta} \hat{\gamma}}$ and $N_{\alpha \hat{\beta} \hat{\gamma}}$ will remain unused in the remainder of this work we defer an explicit display of these quantities into appendix A. Inserting (5) into (2) we arrive at the Hamiltonian

$$
\begin{aligned}
H_{B B}= & \frac{a}{2} D+H_{0}+H_{1}+H_{2}+H_{3}+H_{4} \\
& +\sum_{l} \lambda_{l}\left(s_{l}^{\dagger} s_{l}+t_{l \alpha}^{\dagger} t_{l \alpha}+q_{l \alpha}^{\dagger} q_{l \alpha}-1\right) \\
H_{0}= & \sum_{l}\left(-2 s_{l}^{\dagger} s_{l}-t_{l \alpha}^{\dagger} t_{l \alpha}+q_{l \alpha}^{\dagger} q_{l \alpha}\right) \\
H_{1}= & -\frac{2 b}{3} \sum_{l}\left(t_{l \alpha}^{\dagger} t_{l+1 \alpha} s_{l+1}^{\dagger} s_{l}+t_{l \alpha}^{\dagger} t_{l+1 \alpha}^{\dagger} s_{l+1} s_{l}+h . c .\right) \\
H_{2}= & \frac{b}{\sqrt{6}} \sum_{l}\left(i \varepsilon_{\alpha \beta \gamma} t_{l+1 \alpha}^{\dagger} t_{l \beta}^{\dagger} t_{l \gamma} s_{l+1}+h . c .\right) \\
H_{3}= & -\frac{b}{4} \sum_{l}\left(t_{l \alpha}^{\dagger} t_{l+1 \alpha}^{\dagger} t_{l+1 \beta} t_{l \beta}-t_{l \alpha}^{\dagger} t_{l+1 \beta}^{\dagger} t_{l+1 \alpha} t_{l \beta}\right) \\
H_{4}= & O\left(q^{(\dagger)}\right)
\end{aligned}
$$

where $\lambda_{l}$ is a local Lagrange multiplier to enforce the constraint (伍). $H_{4}$ refers to quartic terms involving at least one quintet and at most one singlet boson. Note that the local Hamiltonian $H_{0}$ and the first term $\mathrm{Da} / 2$ simply reflects the spectrum of the single tetrahedron.

To treat the interacting bose system (6) approximations have to be made. To this end we first realize that in the limit of weak inter-tetrahedral coupling, i.e. $b \ll 1$, the singlet bosons will condense1 1 , to with $s_{l}^{(\dagger)} \rightarrow s \in \Re$. Focusing on this limit and keeping only terms up to quadratic order in the boson operators and, moreover, replacing the local Lagrange multiplier $\lambda_{l}$ by a global one we arrive at the mean-field theory (MFT)

$$
H_{M F T}=D\left(-2 s^{2}+\lambda s^{2}-\lambda+\frac{a}{2}\right)
$$




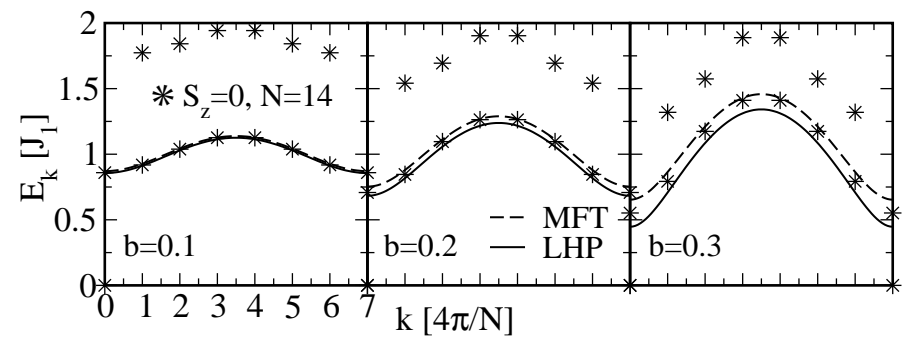

FIG. 4. Dashed (solid) line: $E_{k}$ as from (10) for MFT (LHP). Stars: first two total- $S_{z}=0$ excitation of dimerized spin-1 chain from ED with PBC.

$$
\begin{aligned}
+\sum_{l \alpha}(\lambda+1) q_{l \alpha}^{\dagger} q_{l \alpha}-\frac{1}{2} \sum_{k \alpha}(\lambda-1) \\
+\frac{1}{2} \sum_{k \alpha} \Psi_{k \alpha}^{\dagger}\left[\begin{array}{cc}
\lambda-1+s^{2} \epsilon_{k} & s^{2} \epsilon_{k} \\
s^{2} \epsilon_{k} & \lambda-1+s^{2} \epsilon_{k}
\end{array}\right] \Psi_{k \alpha} \\
\epsilon_{k}=-\frac{4}{3} b \cos (k)
\end{aligned}
$$

where $D$ is the number of dimers and $k$ is a momentum vector. $\Psi_{k \alpha}^{(\dagger)}$ is a a spinor with $\Psi_{k \alpha}^{\dagger}=\left[t_{k \alpha}^{\dagger} t_{-k \alpha}\right]$ and $t_{l \alpha}^{\dagger}=\sum_{k} e^{-i k l} t_{k \alpha}^{\dagger} / \sqrt{D}$. The mean-field Hamiltonian can be diagonalized by a Bogoliubov transformation yielding

$$
\begin{aligned}
& H_{M F T}=D\left(\frac{3}{2}-2 s^{2}+\lambda s^{2}-\frac{5}{2} \lambda+\frac{a}{2}\right) \\
& +\sum_{l \alpha}(\lambda+1) q_{l \alpha}^{\dagger} q_{l \alpha}+\sum_{k \alpha} E_{k}\left(a_{k \alpha}^{\dagger} a_{k \alpha}+\frac{1}{2}\right),
\end{aligned}
$$

where the threefold degenerate triplet energy $E_{k}$ is given by

$$
E_{k}=\sqrt{(\lambda-1)^{2}\left(1+\frac{s^{2}}{\lambda-1} 2 \epsilon_{k}\right)}
$$

and the Bogoliubons $a_{k \alpha}^{(\dagger)}$ result from

$$
\Psi_{k \alpha}=\left[\begin{array}{cc}
g_{k} & h_{k} \\
h_{k} & g_{k}
\end{array}\right] \Phi_{k \alpha}
$$

where $\Phi_{k \alpha}^{(\dagger)}$ is a a spinor with $\Phi_{k \alpha}^{\dagger}=\left[\begin{array}{ll}a_{k \alpha}^{\dagger} & a_{-k \alpha}\end{array}\right]$ and $h_{k}^{2}=\left[\left(1+\epsilon_{k}\right) / E_{k}-1\right] / 2$, and $h_{k} g_{k}=-\epsilon_{k} /\left(2 E_{k}\right)$ with $h_{k}^{2}-g_{k}^{2}=1$. Note that on the quadratic level the quintet is dispersionless Substituting $d=s^{2} /(\lambda-1)$ the groundstate energy is 22

$$
\begin{gathered}
E_{M F T}^{0}=D\left(\frac{3}{2}-2 s^{2}+\lambda s^{2}-\frac{5}{2} \lambda+\frac{a}{2}\right) \\
+\frac{3}{2}(\lambda-1) \sum_{k} \sqrt{1+2 d \epsilon_{k}}
\end{gathered}
$$

where we have used that $\left\langle t_{l \alpha}^{\dagger} t_{l \alpha}\right\rangle=\left\langle q_{l \alpha}^{\dagger} q_{l \alpha}\right\rangle=0$ in the gaped case at $T=0$. The mean-field order parameters $s^{2}$ and $\lambda$ follow from the saddlepoint conditions $\partial E_{M F T}^{0} / \partial s^{2}=0$ and $\partial E_{M F T}^{0} / \partial \lambda=0$ which can be combined to result in

$$
\begin{aligned}
& \frac{5}{2}-d-\frac{3}{2 D} \sum_{k} \frac{1}{\sqrt{1+2 d \epsilon_{k}}}=0 \\
& \lambda-2+\frac{3}{2 D} \sum_{k} \frac{\epsilon_{k}}{\sqrt{1+2 d \epsilon_{k}}}=0,
\end{aligned}
$$

with (13) independent of $\lambda$. Therefore, only the single selfconsistency equation (13) has to be solved for $d$ with $\lambda$ following from direct insertion of $d$ into (14).

In the limit of vanishing inter-tetrahedral coupling, i.e. $b=0(13,14)$ reduce to

$$
d=1 \quad, \quad \lambda=2 \quad \rightarrow \quad s^{2}=1 .
$$

This case relates the MFT to the linearized HolsteinPrimakoff (LHP) method 19,20, which has found frequent use in bond-boson approaches to dimerized spin- $1 / 2$ systems. Within the LHP the constraint is used to eliminate the singlets on the tetrahedra, i.e. within $H_{0}$, by $s_{l}^{\dagger} s_{l}=1-t_{l \alpha}^{\dagger} t_{l \alpha}-q_{l \alpha}^{\dagger} q_{l \alpha}$. Moreover, within $H_{1, \ldots, 4}$ the singlet condensation is implemented with unit strength, i.e., $s_{l}^{(\dagger)}=1$. Dropping all terms beyond quadratic order in the boson operators we arrive at a Hamiltonian which is exactly identical to (7) with however $\lambda \equiv 2$ and $s^{2} \equiv 1$. Therefore the LHP is identical to the MFT constrained to (15). A priori it is not obvious whether the MFT or the LHP is a more reliable approximation and we will present results obtained from both methods.

In fig. 2 results for $a_{c}(b)$ as obtained from (12) are included for the MFT and LHP approach. At the dimer to singlet-product phase-boundary the agreement with ED is very good, both for LHP and MFT. In principle, the singlet condensate restricts the bond-boson approaches to the dimer phase. In fact, the LHP spin-gap closes at $b=3 / 8$ confining the LHP to $b<3 / 8<b_{c}$. The MFT can be continued from the dimer into the Haldane regime, even though the ground-state symmetries are different, yielding a transition line qualitatively still comparable to ED.

Next we consider elementary excitations of the dimer state. These may (i) remain in the dimerized spin-1 chain sector, or (ii) involve transitions into sectors containing localized edge-singlets, i.e. sites with $T_{i l}=0$. In this paper we confine ourselves to the former type. As has been pointed out in ref.8, for a single tetrahedron, the energy of a type-(ii) $\mathcal{S}_{2}$-excitation resides within the spin-gap of the type-(i) excitations for $1 / 2 \leq a \leq 1$. Analogous dispersionless singlet gap-states occur in the spin gap of the dimer phase of the lattice model and will be discussed elsewhere 23. Figure 4 compares the dispersion obtained from (10), both for the MFT and LHP for various values of $b<b_{c}$, with the first two $S_{z}=0$ eigenstates obtained from $\mathrm{ED}$ on a finite dimerized spin-1 chain with PBC. Regarding the first triplet excitations the agreement is very good. A comparison of the spin gap, i.e. $E_{k=0, \pi}$, as 
obtained from the MFT and LHP approach in the dimerized spin-1 chain sector with ED is contained in fig. 3 . Apart from the fact that the agreement is satisfactory for $b \lesssim 0.2$ this figure demonstrates the main difference between the MFT and LHP approximation. In contrast to the LHP spin-gap which closes for $b>3 / 8$ the MFT overestimates the binding energy due to dimer formation and keeps the spin gap opened for all values of $b$.

\section{TWO-TRIPLET EXCITATIONS AND RAMAN SCATTERING}

Raman scattering can be used to probe the total-spin zero excitations of a spin system at zero momentum. In this section we consider the magnetic Raman scattering in the dimerized $S=1$ sectpr of the tetrahedral chain. Following Loudon and Fleury 24 the Raman scattering operator is given by

$$
\begin{aligned}
R & =\sum_{l m} a_{l m}\left(\mathbf{E}_{i} \cdot \mathbf{n}_{l m}\right)\left(\mathbf{E}_{o} \cdot \mathbf{n}_{l m}\right) \mathbf{s}_{l} \cdot \mathbf{s}_{m} \\
& =A E_{i} E_{o} \sum_{l}\left(\mathbf{T}_{l 1} \mathbf{T}_{l 2}+\beta \mathbf{T}_{l 2} \mathbf{T}_{l+11}\right)
\end{aligned}
$$

$\mathbf{E}_{i(o)}$ are the incoming(outgoing) electric-field vectors and $\mathbf{n}_{l m}$ are unit vectors connecting exchange-coupled sites. $a_{l m}$ are matrix element which are identical among each of those exchange paths corresponding to one of $J_{1}$, $J_{2}$, or $J_{3}$. From this and the geometry of the tetrahedral chain the second line results for polarizations of the light along the chain - which we will focus on. While $\beta$ in (16) will be of order $b$, it is very unlikely that $\beta=b$. In the latter case the Raman operator commutes with the Hamiltonian implying a vanishing Raman intensity at nonzero Raman shifts. In the former case we use an equivalent Raman operator

$$
\tilde{R}=R-\left.R\right|_{\beta=b}=C \sum_{l} \mathbf{T}_{l 2} \mathbf{T}_{l+11},
$$

where $C=A E_{i} E_{o}(\beta-b)$. Thus the Raman intensity will be of second order in $\beta$ and $b$, i.e. the inter-tetrahedral coupling. To proceed, we approximate $\tilde{R}$ on the level of the LHP

$$
\begin{aligned}
\tilde{R}_{L H P}:= & \lim _{q \rightarrow 0} \tilde{R}_{L H P}(q)=\lim _{q \rightarrow 0}\left[-\frac{2 C}{3} \sum_{k} \cos (k+q / 2)\right. \\
& \left.\times\left(t_{k+q \alpha}^{\dagger}+t_{-k-q \alpha}\right)\left(t_{-k \alpha}^{\dagger}+t_{k \alpha}\right)\right]
\end{aligned}
$$

where, for later convenience, we have introduced an auxiliary momentum dependence. The Raman intensity can be obtained from the zero momentum limit of the dynamical susceptibility

$$
\chi(q, \tau)=\left\langle T_{\tau}\left[\tilde{R}_{L H P}(q, \tau) \tilde{R}_{L H P}(q, 0)\right]\right\rangle .
$$

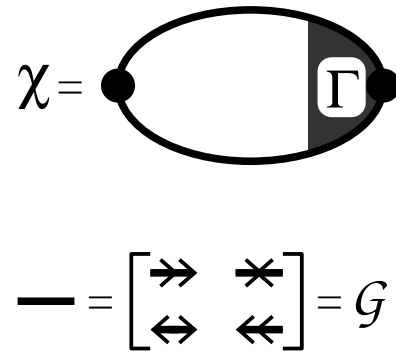

$$
=\widetilde{\mathrm{R}}_{\mathrm{LHP}}
$$

FIG. 5. Raman susceptibility. Thick solid lines label the dressed, $2 \times 2$ one-triplet, i.e. $t^{(\dagger)}$-particle matrix Greens-functions including diagonal and anomalous contributions. The solid dot refers to the Raman operator (18). $\Gamma$ is the two-triplet reducible vertex.

Since $\chi(q, \tau)$ is a two-particle propagator, it is important to assess the relevance of two-particle scattering. In particular, it has been realized in the context of other dimerized spin-1/2 systems that magnetic bound states can severely renormalize the bare two-triplet spectrum 2528.

We chose to implement the two-particle scattering within the LHP approach. Apart from the interactions $H_{2 \ldots 4}$ in (6) the constraint (4) implies a hard-core repulsion between two bosons on a site. In the LHP this pertains only to the triplets, as the singlets are condensed and the quintets have been discarded. The hard-core is incorporated directly by introducing an additional contribution to the Hamiltonian 25

$$
H_{U}=U \sum_{l} t_{l \alpha}^{\dagger} t_{l \beta}^{\dagger} t_{l \alpha} t_{l \beta}
$$

with the summation convention on the Greek indices. $\chi(q, \tau)$ is evaluated with $H_{U}$ at finite $U$ and the limit of $U \rightarrow \infty$ is taken at the end.

The Raman susceptibility corresponds to the diagram depicted in fig. 5. To simplify matters we focus on the limit $b \ll 1$. In that limit the ground state is nearly a pure product of $\mathcal{S}_{1}$ singlets and the triplet density induced by quantum fluctuations $n_{t}=\left\langle t_{l \alpha}^{\dagger} t_{l \alpha}\right\rangle=3 \sum_{k} h_{k}^{2}$ is a small parameter. As a consequence only the twotriplet pair-creation(destruction) vertices contained in (18) are relevant. Moreover, contributions to the reducible two-particle propagator in fig. 5 involving anomalous Greens functions, as well as one-triplet selfenergy insertions, are suppressed by factors of the triplet density and will be neglected25. Physically speaking, the Stokes Raman-process creates two triplets within an approximate singlet product-state. These propagate along the tetrahedral chain and form an interacting two-particle problem with no additional triplets generated(destroyed) by quantum fluctuations27.

The two-triplet problem allows for an exact solution 
a)

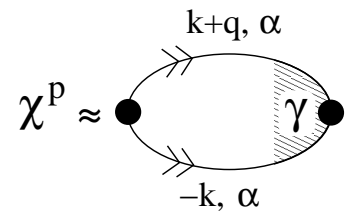

b)

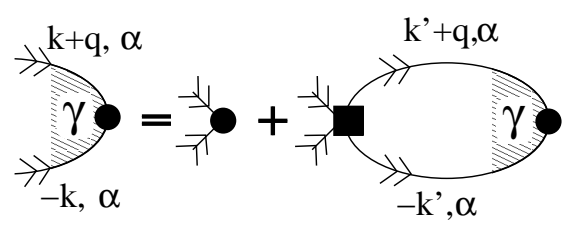

c)
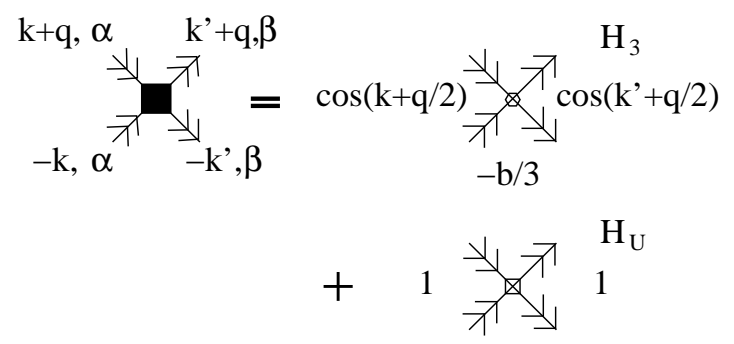

$2 \mathrm{U} / 3$

FIG. 6. T-matrix approximation to fig. 5: thin, doubly-directed lines label 11-elements of the bare one-triplet Greens function (21). The solid dot is the two-triplet part of the Raman vertex (18). Summation on $k^{\prime}$ and $\alpha$ is implied in all bare triplet-bubbles. $\gamma($ solid square) refers to two-triplet (ir)reducible vertex. Analytic expressions for the two irreducible vertices due to $H_{3}$ and $H_{U}$ are displayed incorporating all possible leg exchanges.

including $H_{U}$ by the T-matrix approach of fig. 6. This figure depicts the 'particle-diagrams', which correspond to the Stokes process. For the Anti-Stokes process an identical set of 'hole-diagrams' exists with all lines reversed. In the singlet channel only $H_{3}$ of (6) and $H_{4-\text { contribute }}$ to the irreducible two-particle vertex $\gamma(\sec 29)$. Due to the momentum space symmetry of $\gamma$ it is convenient to formulate the T-matrix equation using a $2 \times 2$ matrix notation. The bare one-triplet Greens function including normal and anomalous components is given by

$$
\begin{aligned}
G_{\alpha}^{i j}\left(k, i \omega_{n}\right) & =\frac{1}{\left(i \omega_{n}\right)^{2}-E_{k}^{2}} \\
& \times\left[\begin{array}{cc}
i \omega_{n}+1+\epsilon_{k} & -\epsilon_{k} \\
-\epsilon_{k} & -i \omega_{n}+1+\epsilon_{k}
\end{array}\right],
\end{aligned}
$$

where $\epsilon_{k}$ and $E_{k}$ are as of (8, 10,15), $\alpha=x, y, z$, and $\omega_{n}=$ $2 n \pi T . G_{\alpha}^{i j}\left(k, i \omega_{n}\right)$ satisfies the symmetries $G_{\alpha}^{11}\left(k, i \omega_{n}\right)=$ $G_{\alpha}^{22}\left(k,-i \omega_{n}\right)$ and $\left[G_{\alpha}^{21}\left(k, i \omega_{n}\right)\right]^{*}=G_{\alpha}^{21}\left(k, i \omega_{n}\right)$. From fig. 6 we get

$$
\begin{aligned}
& \chi^{p}(q, z)=2 \chi_{c c}^{0} \\
& +2\left[\chi_{c c}^{0} \chi_{c 1}^{0}\right] \mathbf{V}\left\{\mathbf{1}-\left[\begin{array}{cc}
\chi_{c c}^{0} & \chi_{c 1}^{0} \\
\chi_{1 c}^{0} & \chi_{11}^{0}
\end{array}\right] \mathbf{V}\right\}^{-1}\left[\begin{array}{c}
\chi_{c c}^{0} \\
\chi_{1 c}^{0}
\end{array}\right],
\end{aligned}
$$

where $\chi_{c c, c 1,1 c, 11}^{0}$ are bare two-particle propagators, the explicit display of whose momentum and frequency dependence has been suppressed for brevity. V incorporates the momentum independent coupling-constant factors of the two vertices in $\gamma$ of fig. 6 .

$$
\begin{aligned}
& \mathbf{V}=\left[\begin{array}{ll}
-b / 3 & 2 U / 3
\end{array}\right] \\
& \chi_{11}^{0}(q, z)=3 \sum_{k} \frac{1}{z-E_{k+q}-E_{k}} \stackrel{b \ll 1}{\simeq} g A \\
& \chi_{1 c}^{0}(q, z)=3 \sum_{k} \frac{\cos \left(k+\frac{q}{2}\right)}{z-E_{k+q}-E_{k}} \stackrel{b \ll 1}{\simeq} g(1-\nu A) \\
& \chi_{c c}^{0}(q, z)=3 \sum_{k} \frac{\cos ^{2}\left(k+\frac{q}{2}\right)}{z-E_{k+q}-E_{k}} \stackrel{b \ll 1}{\cong}-g \nu(1-\nu A),
\end{aligned}
$$

where we have analytically continuated $i \omega_{n}$ into the upper complex plane $i \omega_{n} \rightarrow z$ and have restricted ourselves to the zero temperature limit. The prefactors of 3 are due to the sum over the triplet index $\alpha$ and $\chi_{1 c}^{0}(q, z)=\chi_{c 1}^{0}(q, z)$. The two-hole propagator $\chi^{h}$ is obtained by reversing the signs of all $E_{(k) k+q}$ in the denominators of (24). In the limit $b \ll 1$ one may expand the square root in (10) which allows for analytic expressions for all of the $\chi^{0}$ s in terms of the quantities $g, \nu$ and $A$

$$
\begin{aligned}
& g=g(q)=\frac{9}{8 b \cos (q / 2)} \quad \nu=\nu(q, z)=\frac{3(z-2)}{8 b \cos (q / 2)} \\
& A=A(q, z)=\frac{\operatorname{sign}(\operatorname{Re}(\nu))}{\sqrt{\nu^{2}-1}} .
\end{aligned}
$$

From (22,25) we obtain the Stokes susceptibility from $\chi^{p}(q, z)$ by performing the limit $U \rightarrow \infty$

$$
\begin{aligned}
& \chi^{p}(q, z)=\frac{6\left[\chi_{11}^{0} \chi_{c c}^{0}-\left(\chi_{1 c}^{0}\right)^{2}\right]}{3 \chi_{11}^{0}+b\left[\chi_{11}^{0} \chi_{c c}^{0}-\left(\chi_{1 c}^{0}\right)^{2}\right]} \\
& \stackrel{b \ll 1}{\simeq} \frac{6\left(\operatorname{sign}(\operatorname{Re}(\nu)) \sqrt{\nu^{2}-1}-\nu\right)}{b\left[\operatorname{sign}(\operatorname{Re}(\nu)) \sqrt{\nu^{2}-1}-\nu-8 \cos (q / 2) / 3\right]} .
\end{aligned}
$$

As in (22) we refrain from explicitly displaying the momentum and frequency dependence on the r.h.s. of $(26)$. From (26,27) one obtains the Raman intensity $I(\omega)$ from $I(\omega)=-\operatorname{Im} \chi^{p}\left(0, z \rightarrow \omega+i 0^{+}\right)$where $\omega$ refers to the Raman shift.

Figure 7 shows the Raman intensity contrasting the bare two-triplet spectrum with the interacting one. As is obvious the bare intensity is strongly renormalized by the two-triplet interactions. In particular, both of the vanHove singularities present in the bare two-triplet spectrum disappear with the almost symmetric shape of the bare spectrum being deformed by a downward shift of the intensity. These findings allow for a clear physical interpretation which follows from an inspection of the denominators of (2627). For $q>q_{c}$ these denominators acquire a zero for energies $E_{B}(q)$ below the continuum 


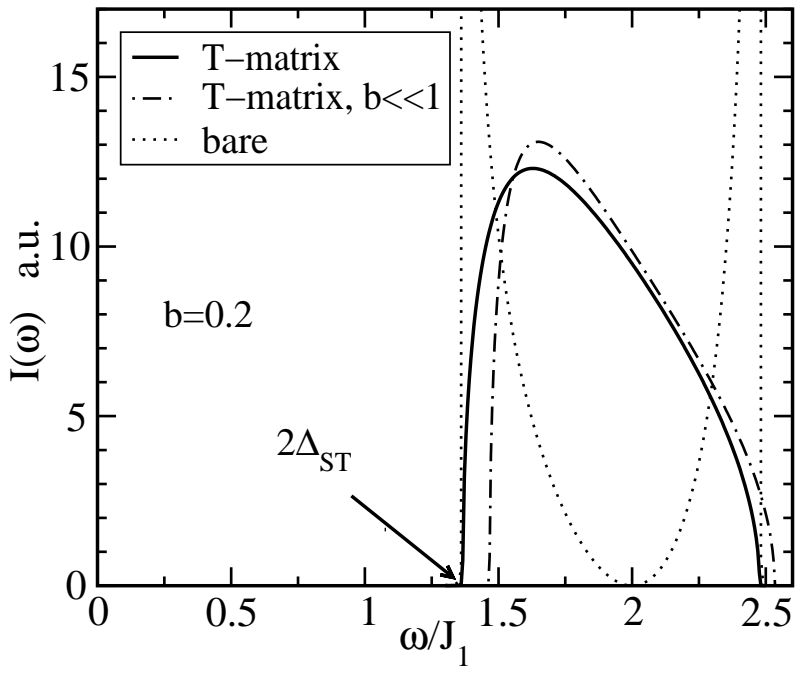

FIG. 7. Raman intensity in the dimer phase at $b=0.2$ as obtained from 26) (solid), from (27) dashed-dotted, and from (22) for $\mathbf{V}=0$ (dotted) line. $2 \Delta_{S T}$ refers to twice the singlet-triplet gap.

of the two-triplet scattering states. I.e., a total-spin zero bound state exists in the dimerized spin-one chain at finite momentum 30 . Figure 8 shows the dispersion of this bound state as obtained from (27) where $q_{c}=2 \operatorname{acos}(3 / 8)$. For $q<q_{c}$ the bound state turns into a resonance shortly above the lower edge of the continuum which leads to the asymmetric Raman intensity of fig. I at $q=0$. This resonance feature has to be contrasted with the impact of bound states on the Raman spectra of other dimerized and frustrated low-dimensional quantum spin systems where $S=0$ collective modes have beepobserved rather as sharp excitations within the spin gap 32 34. The actual location of the bound sate with respect to the twotriplet continuum is significantly affected by the hardcore repulsion $U$. Setting $U=0$ in (22) the short-range attraction due to $H_{3}$ would be overestimated with $E_{B}(q)$ resulting from $1+b \chi_{c c}^{0}\left(q, E_{B}(q)\right) / 3=0$ which would yield a bound state below the lower edge of the continuum for all $q$. While $E_{B}(q)$ in fig. 8 has been plotted in units of $J_{1}$ for $b=0.2$ all bound state dispersions can be rescaled onto a single one in terms of the frequency variable $\nu$. This is certainly an artifact of the limit $b \ll 1$. Finally we note that the relative agreement between (26) and (27) improves continuously as $b \rightarrow 0$.

\section{CONCLUSION}

In summary we have investigated the ground state and several aspects of the one- and two-triplet excitations of a tetrahedral cluster spin-chain. A number of open questions remain. In particular excitations involving localized edge singlets of the tetrahedra are an issue yet to be resolved. In the case that such excitations are Ra-

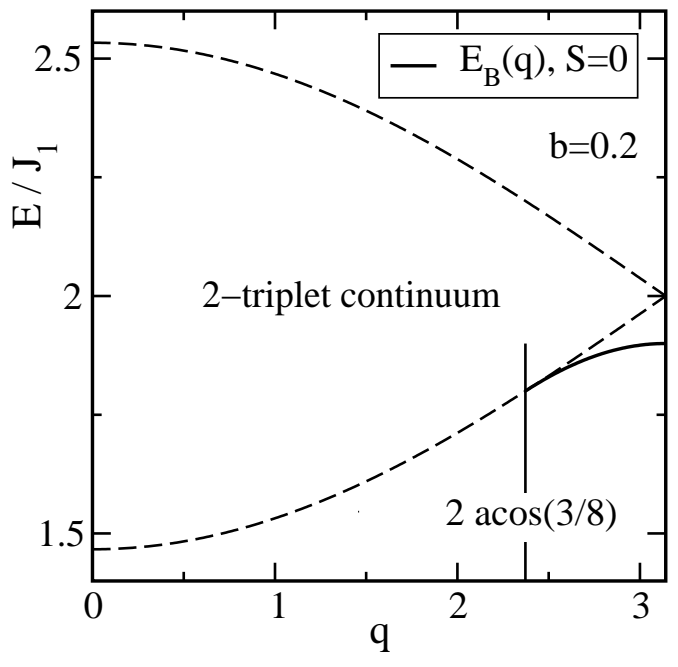

FIG. 8. Two-triplet continuum and dispersion of the $S=0$ bound state from (27). Note the y-axis offset.

man active we expect them to lead to a dispersionless distribution of intensities which can reside in the spingap of fig. 7 for certain ranges of the parameters $(a, b)$. Below a temperature of $T \lesssim 50 \mathrm{~K}$ the Raman intensity on $\mathrm{Cu}_{2} \mathrm{Te}_{2} \mathrm{O}_{5} \mathrm{Br}_{2}$ gradually builds up a continuum $\mathrm{g}$ centered at $60 \mathrm{~cm}^{-1}$ which, below $T \lesssim 8 \mathrm{~K}$ is accompanied by an additional sharp mode developing at $20 \mathrm{~cm}^{-1}$. One might speculate the continuum to correspond to that of fig. 17 and the sharp mode to consist of transitions involving edge-singlets. Yet, the measured continuum is rather more symmetric than the solid line in fig. 7. This might be related to the effects of three-dimensional couplings between the tetrahedra in the tellurates leaving their magnetism an open issue which deserves further studies. Finally the role of perturbations breaking the complete frustration may be of relevance in the vicinity of the first-order transition leading to additional quantum phases.

Acknowledgments: It is a pleasure to thank $\mathrm{P}$. Lemmens, R. Valenti, C. Gros, F. Mila, E. Kaul, and Ch. Geibel for stimulating discussions and comments. This research was supported in part by the Deutsche Forschungsgemeinschaft under Grant No. BR 1084/1-1 and BR 1084/1-2 and trough SFB 463.

\section{APPENDIX A: $\mathcal{T}_{1} \leftrightarrow \mathcal{Q}$ AND $\mathcal{Q} \leftrightarrow \mathcal{Q}$ TRANSITION MATRIX ELEMENTS}

In table (III IV) of this appendix we list the nonzero matrix elements of $M_{\alpha \hat{\beta} \hat{\gamma}}$ and $N_{\alpha \hat{\beta} \hat{\gamma}}$ from (5). One should note, that there are no transitions mediated by $S_{l 1,2}^{\alpha}$ between $\mathcal{S}_{1}$ and $\mathcal{Q}$. 


\begin{tabular}{ccc|ccc|ccc}
\hline \hline$\hat{\beta}$ & $\hat{\gamma}$ & $2^{3 / 2} M_{x \hat{\beta} \hat{\gamma}}$ & $\hat{\beta}$ & $\hat{\gamma}$ & $2^{3 / 2} M_{y \hat{\beta} \hat{\gamma}}$ & $\hat{\beta}$ & $\hat{\gamma}$ & $2^{3 / 2} M_{z \hat{\beta} \hat{\gamma}}$ \\
\hline 2 & 5 & 1 & 2 & 5 & $i$ & & & \\
2 & 7 & $-\sqrt{\frac{2}{3}}$ & 2 & 9 & $-i$ & 2 & 6 & -1 \\
2 & 9 & 1 & 3 & 5 & -1 & 2 & 8 & 1 \\
3 & 5 & $i$ & 3 & 7 & $-\sqrt{\frac{2}{3}}$ & 3 & 6 & $-i$ \\
3 & 9 & $-i$ & 3 & 9 & -1 & 3 & 8 & $-i$ \\
4 & 6 & -1 & 4 & 6 & $-i$ & 4 & 7 & $2 \sqrt{\frac{2}{3}}$ \\
4 & 8 & 1 & 4 & 8 & $-i$ & & & \\
\hline \hline
\end{tabular}

TABLE III. Nonzero transition-operator matrix-elements, see (5), conecting $\mathcal{T}_{1}$ and $\mathcal{Q}$ as of table II.

\begin{tabular}{|c|c|c|c|c|c|c|c|c|}
\hline$\hat{\beta}$ & $\hat{\gamma}$ & $2 N_{x \hat{\beta} \hat{\gamma}}$ & $\hat{\beta}$ & $\hat{\gamma}$ & $2 N_{y \hat{\beta} \hat{\gamma}}$ & $\hat{\beta}$ & $\hat{\gamma}$ & $2 N_{z \hat{\beta} \hat{\gamma}}$ \\
\hline 5 & 6 & 1 & 5 & 6 & $-i$ & \multirow{8}{*}{$\begin{array}{l}5 \\
6 \\
8 \\
9\end{array}$} & \multirow{8}{*}{$\begin{array}{l}5 \\
6 \\
8 \\
9\end{array}$} & \multirow{8}{*}{$\begin{array}{c}2 \\
1 \\
-1 \\
-2\end{array}$} \\
\hline 6 & 5 & 1 & 6 & 5 & $i$ & & & \\
\hline 6 & 7 & $\sqrt{\frac{3}{2}}$ & 6 & 7 & $-i \sqrt{\frac{3}{2}}$ & & & \\
\hline 7 & 6 & $\sqrt{\frac{3}{2}}$ & 7 & 6 & $i \sqrt{\frac{3}{2}}$ & & & \\
\hline 7 & 8 & $\sqrt{\frac{3}{2}}$ & 7 & 8 & $-i \sqrt{\frac{3}{2}}$ & & & \\
\hline 8 & 7 & $\sqrt{\frac{3}{2}}$ & 8 & 7 & $i \sqrt{\frac{3}{2}}$ & & & \\
\hline 8 & 9 & 1 & 8 & 9 & $-i$ & & & \\
\hline 9 & 8 & 1 & 9 & 8 & $i$ & & & \\
\hline
\end{tabular}

TABLE IV. Nonzero transition-operator matrix-elements, see (5), within $\mathcal{Q}$ as of table III.

${ }^{1}$ E. Müller-Hartmann, R.R.P. Singh, C. Knetter, and G. S. Uhrig, Phys. Rev. Lett. 84, 1808 (2000).

${ }^{2}$ H. Kageyama, K. Yoshimura, R. Stern, N. V. Mushnikov, K. Onizuka, M. Kato, K. Kosuge, C. P. Slichter, T. Goto, and Y. Ueda, Phys. Rev. Lett. 82, 3168 (1999).

${ }^{3}$ S. Miyahara and K. Ueda, Phys. Rev. Lett. 82, 3701 (1999).

${ }^{4}$ B.S. Shastry and B. Sutherland, Physica 108B, 1069 (1981).

${ }^{5}$ I. Bose and S. Gayen, Phys. Rev. B 48, 10653 (1993).

${ }^{6}$ A. Ghosh and I. Bose, Phys. Rev. B 55, 3613 (1997).

${ }^{7}$ A. Honecker, F. Mila, M. Troyer, Eur. Phys. J. B. 15, 227 (2000).

${ }^{8}$ M. Johnsson, K.W. Törnross, F. Mila, and P. Millet, Chem. Mater. 12, 2853 (2000).

${ }^{9}$ P. Lemmens, et al., to appear in: Advances in Solid State Physics (2001).

${ }^{10}$ We have checked $e(b, N)$ to increase monotonously with $N$ by evaluating the ground-state energy per site of a dimerized $S=1$ chain with open boundary conditions as a function of system size.

11 T. Sakai and M. Takahashi Phys. Rev. B 43, 13383 (1991).

12 S.R. White and D.A. Huse, Phys. Rev. B 48, 3844 (1993).

13 O. Golinelli, T. Jolicoeur, and R. Lacaze, Phys. Rev. B 50, 3037 (1994).

${ }^{14}$ Y. Kato and A. Tanaka, J. Phys. Soc. Jp. 63, 1277 (1993)

${ }^{15}$ K. Totsuka, Y. Nishiyama, N. Hatano, and M. Suzuki, J. Phys. Condensed Matter 74895 (1995).

16 S. Sachdev and R. N. Bhatt, Phys. Rev. B 41, 9323 (1990).

17 A. V. Chubukov and Th. Jolicoeur, Phys. Rev. B 44, 12050 (1991).

18 S. Gopalan, T. M. Rice, M. Sigrist, Phys. Rev. B 49, 8901 (1994).
${ }^{19}$ O. A. Starykh, M. E. Zhitomirsky, D. I. Khomskii, R. R. P. Singh, and K. Ueda, Phys. Rev. Lett. 77, 2558 (1996).

${ }^{20}$ R. Eder, Phys. Rev. B 57, 12832 (1998).

${ }^{21}$ W. Brenig, Phys. Rev. B 56, 14441 (1997).

${ }^{22}$ Only the case of $\lambda>1$ occurs in physically relevant solutions of the MFT.

${ }^{23} \mathrm{~W}$. Brenig and K.W. Becker, to be published elsewhere.

24 P.A. Fleury and R. Loudon, Phys. Rev. 166, 514 (1968).

${ }^{25}$ O.P. Sushkov and V.N. Kotov Phys. Rev. Lett. 81, 1941 (1998)

${ }^{26}$ V. N. Kotov, O. P. Sushkov, and R. Eder, Phys. Rev. B, 6266 (1999).

${ }^{27}$ C. Jurecka and W. Brenig Phys. Rev. B 61, 14307 (2000).

${ }^{28}$ S. Trebst,H. Monien, C.J. Hamer,Z. Weihong, and R.R.P. Singh, Phys. Rev. Lett. 85, 4373 (2000)

${ }^{29}$ In principle two-particle irreducible vertices can be constructed to second order in $b$ from $\mathrm{H}_{2}$. In the singlet channel, however, because of the symmetries of the spin indices in (18) w.r.t. those of the Levi-Civita symbol in $\mathrm{H}_{2}$ these vanish identically.

${ }^{30}$ Using a different technique an expression very similar to the denominator of (27) has been obtained in a study of phonon-assistefoptical two-triplet-absorption on spin-1/2 two-leg ladder 27 . In contrast to the dimerized $S=1$ chain case, however, $q_{c}=0$ has been found for the ladder in that case. In that work it was predicted, that the singlet bound-state of the ladder should be observable in optical absorbtion. Recent work has confirmed this prediction 31 .

${ }^{31}$ M. Windt, M. Grueninger, T. Nunner, C. Knetter, K. Schmidt, G. S. Uhrig, T. Kopp, A. Freimuth, U. Ammerahl, B. Buechner, and A. Revcolevschi, cond-mat/0103438.

${ }^{32}$ P. Lemmens, M. Fischer, G. Güntheroth, C. Gros, P. van Dongen, M. Weiden, W. Richter, C. Geibel, and F. Steglich, Phys. Rev. B 55, 15076 (1997).

${ }^{33}$ P. Lemmens, M. Fischer, G. Els, G. Güntherodt, A.S. Mishchenko, M. Weiden, R. Hauptmann, C. Geibel, and F. Steglich, Phys. Rev. B 58, 14159 (1998).

${ }^{34}$ P. Lemmens, M. Grove, M. Fischer, G. Güntherodt, V.N. Kotov, H. Kageyama, K. Onizuka, and Y. Ueda, Phys. Rev. Lett. 85, 2605 (2000). 